\title{
Active Tensegrity: a control framework for an adaptive
}

\section{civil engineering structure}

\author{
Bernard Adam ${ }^{1}$ and Ian F.C. Smith ${ }^{2}$ \\ ${ }^{1} \mathrm{PhD}$, Ingphi SA, Consulting Engineers, Place St-François 2, 1003 Lausanne, Switzerland. \\ E-mail: bernard.adam@a3.epfl.ch \\ ${ }^{2}$ Professor, Structural Engineering Institute, Ecole Polytechnique Fédérale de Lausanne \\ (EPFL), Station 18, 1015 Lausanne, Switzerland. E-mail: ian.smith@epfl.ch
}

\begin{abstract}
This paper presents progress in the field of adaptive civil-engineering structures. Selfdiagnosis, multi-objective shape control and reinforcement-learning processes are implemented within a control framework on an active tensegrity structure. Self-diagnosis extends active structural control to situations of partially defined loads. Multi-objective search is useful for computing commands that control shape while minimizing active strut stroke and stress, and maximizing stiffness. Reinforcement learning improves the control by memorizing, retrieving and adapting previous control events. The control framework is validated experimentally on an active tensegrity structure. This provides an example of an adaptive civil-engineering structure.
\end{abstract}

Keywords: space structures, adaptive civil-engineering structures, tensegrity structures. 


\section{Introduction}

Most civil-engineering structures are passive and static. A more challenging functionality for civil-engineering structures is active adaptation to changing requirements, such as load modifications, temperature variations, support settlements and possible damage occurrence. For the purposes of this paper, the terminology that is related to this field is clarified as follows:

- The concept of active structures as defined by Soong and Manolis (1987) involves structures that include both static and active structural elements.

- Adaptive structures are defined by Wada and Das (1991) to be structures whose performance is controlled by a system composed of sensors, actuators and a computer that provides the ability to learn and improve response to changing environments.

- The ultimate goal of intelligent structures is to maintain and improve structural performance by recognizing changes in behavior and loading, adapting the structure to meet performance goals, and using past events to improve future performance (Shea and Smith 1998, Shea et al. 2002).

The border between the definition of adaptive structures and that of intelligent structures is not clear. The term, adaptive structure, will be used throughout this document.

The words, kinetic architecture, were first employed by Zuk (1968). Large buildings that move themselves to new locations, structures that expand and contract to adapt to changing spatial needs, buildings that change shape and large structures that fold up and are 
transported were discussed. Yao (1972) first introduced active structural control as a means for extending the limits on heights of tall buildings by actively controlling structures during high winds. The behavior of such controlled structures should vary automatically in accordance with unanticipated variations in the loading as well as environmental conditions, thereby producing desirable responses under all possible loading conditions. However, to date, little progress has been found in the literature. Currently, an increasing number of lightweight structures, advances in computing, wireless technology as well as new sensor and actuator technologies create favorable situations for adaptive civil engineering structures. Adaptive civil-engineering structures also provide innovative opportunities for temporary and reusable structures.

While bio-inspired systems have already been studied for decades in domains such as mathematics, informatics, electronics and robotics (Von Neumann 1966, Denning 1976, Teuscher et al. 2003, Floreano and Mondada 1998), few examples of adaptive civil engineering structures have been found in the literature. Pawlowski and Holnicki-Szulc (2004) introduced a structure that could adapt to extreme loads. It detected impacts through a set of sensors and optimally distributed forces in the structure using structural fuses. Sobek et al. (2006) defined adaptive structures as load carrying systems which are able to react to variable external influences. Noak et al. (2006) demonstrated experimentally that integrating actuators in a trussed beam system allows for a significant increase in span and an increased load bearing capacity. However, these are studies where the control command and the structural response are directly linked and not coupled with other control loops. They are carried out mainly on small models and through numerical simulation. Moreover, 
no experimental demonstration of self-diagnosis and self-repair of a civil-engineering structure could be found in the literature.

Since tensegrities can be equipped with active control systems, they have the potential to adapt to their environments. Tensegrities are spatial, reticulated and lightweight structures. These structures are composed of struts and tendons. Stability is provided by the self-stress state between tensioned and compressed elements (Motro 1984). A recent definition has been proposed by Motro (2003): A tensegrity system is a system in a stable selfequilibrated state comprising a discontinuous set of compressed components within a continuum of tensioned components.

Control of tensegrity structures has been a topic of research since the middle of the 1990s. Kawaguchi et al. (1996) studied shape and stress control of pre-stressed truss structures. However, difficulties were identified in validating numerical results through experimental testing. Averseng and Crosnier (2004) studied experimentally the control of a tensegrity grid in which the actuation system is connected to the supports.

Djouadi et al. (1998) described a theoretical scheme to control vibrations of tensegrity systems. Sultan (1999) presented a formulation of tensegrity active control and illustrated it with the example of an aircraft motion simulator. Skelton et al. (2000) concluded that since only small amounts of energy are needed to change the shape of tensegrity structures, they are advantageous for active control. Kanchanasaratool and Williamson (2002) proposed a dynamic model to study tensegrity feedback shape control. Van de Wijdeven and de Jager (2005) studied an example of 2D tensegrity vibration and shape control. These studies 
obtained results mainly from numerical simulation of small, simple and symmetric tensegrity models.

Fest et al. (2004) demonstrated experimentally the feasibility of tensegrity active shape control. The most challenging part of the study was the computation of control commands (sequence of contractions and elongations of active struts) that modify the self-stress state in order to recover the top surface slope of the structure when subjected to a load. Since the behavior of the structure that was used for experimental testing is geometrically nonlinear and highly coupled, there is no closed form solution for active strut adjustments given a required top-surface slope (Fest et al. 2003). This fact precludes the use of analytical treatment such as suggested for example in (Williamson at al. 2003). A single-objective stochastic-search algorithm (Raphael and Smith, 2003) was selected as the best method to accommodate the exponentially complex generate-test process that is needed to compute control commands (Domer et al. 2003). Domer and Smith (2005) studied the capacity of this structure and its control system to learn in order to decrease the average time necessary to compute a control command.

Adam and Smith (2007A) proposed and validated experimentally a multi-objective approach to compute control commands. Since more robust control commands were computed using this approach than with single objective control, the structure was observed to accommodate multiple loading events over its service life. In these studies, it was assumed that the load position and magnitude were known. Adam and Smith (2007B) proposed methodologies of self-diagnosis and self-repair and validated them 
experimentally on an active tensegrity structure. It was concluded that these intelligent control methodologies extend tensegrity active control to situations where loads and damage are partially defined. Control performances were improved by the use of reinforcement learning (Adam and Smith 2008). A general improvement simultaneously in control quality and control command computation rapidity was demonstrated experimentally. However, intelligent-control methodologies were not integrated in a control framework to support active control in partially-defined environments.

No other example of experimentally testing on an adaptive civil engineering structure has been found in the literature. This paper presents an example of an adaptive civil engineering structure using a framework of intelligent-control methodologies: selfdiagnosis (Adam and Smith, 2007B), multi-objective shape control (Adam and Smith 2007A) and reinforcement learning (Adam and Smith, 2008). This framework is integrated in an active tensegrity structure to support control in partially-defined environments.

Following the Section, Description of the active tensegrity structure and the Section Terminology and formulas, the paper is organized as follows. The subsequent section introduces the control framework. The following sections present the active control methodologies: self-diagnosis, multi-objective shape control and reinforcement learning. An example of adaptation over multiple partially defined loads is then given in order to validate the framework experimentally.. The paper ends with a discussion of potential for future work. 


\section{Description of the active tensegrity structure}

The structure that is used for experimental testing is the same as in (Fest et al. 2004). It is composed of 5 modules and rests on three supports (Figure 1). It covers a surface area of $15 \mathrm{~m}^{2}$, has a height of $1.20 \mathrm{~m}$ and withstands a distributed dead load of $300 \mathrm{~N} / \mathrm{m}^{2}$. It is composed of 30 struts and 120 tendons. Struts are fiber-reinforced polymer tubes of 60mm diameter and $703 \mathrm{~mm}^{2}$ cross section. Tendons are stainless-steel cables of $6 \mathrm{~mm}$ in diameter. The central node and star topology is a particularity of each module. This topology was proposed to limit buckling lengths, thereby allowing for more slender compression elements than more traditional tensegrity (Paronesso and Passera 2004). The structure is equipped with an active-control system: ten active struts and three displacement sensors. The ten active struts are placed in-line as pairs in each module. They allow for strut length adjustment. Displacement sensors measure vertical displacements at three top surface nodes: 37, 43 and 48 (Figure 2). Shape control has involved satisfying a serviceability objective: maintaining the slope of the top surface of the structure when the structure was subjected to loading. Top surface slope $S$ is calculated from the vertical coordinates of the top surface three nodes, $Z_{37}, Z_{43}$ and $z_{48}$ that are equipped with displacement sensors (Figure 2). It is determined as follows:

$$
S=\frac{1}{L}\left(z_{43}-\frac{z_{37}+z_{48}}{2}\right)
$$

where $L$ is the horizontal distance between nodes 43 and middle of segment 37 - 48 .

\section{Terminology and Formulas}


For the sake of clarity, notations and quantities that are specific to this structure are introduced. Vertical coordinates of the three nodes 37, 43, 48 are labeled $z_{37}, z_{43}$ and $z_{48}$. Since quantities are measured on the structure and numerically simulated, subscript $m$ is introduced to define measured values and subscript $c$ is introduced to define numerically simulated values. The quantities are introduced below:

- The top surface slope after loading $S^{\prime}$ is evaluated when the structure displaces due to loading. The vertical coordinate of node $i$ in deformed configuration is indicated by $z_{i}^{\prime}$. Consequently the top surface slope after loading $S^{\prime}$ is expressed as follows:

$$
S^{\prime}=\frac{1}{L}\left(z_{43}^{\prime}-\frac{z_{37}^{\prime}+z_{48}^{\prime}}{2}\right)
$$

- The top surface slope deviation TSSD is determined using equations (1) and (2) as follows:

$$
T S S D=S^{\prime}-S
$$

- The top surface slope after active control perturbation $S^{\prime \prime}$ results from a $1 \mathrm{~mm}$ elongation of active struts. The vertical coordinate of node $i$ is labeled $z_{i}$ " and the top surface slope after perturbation $S^{\prime \prime}$ is expressed as follows:

$$
S^{\prime \prime}=\frac{1}{L}\left(z_{43}^{\prime \prime}-\frac{z_{37}^{\prime \prime}+z_{48}^{\prime \prime}}{2}\right)
$$

- The slope variation $\Delta S$ has the following expression using equations (2) and (4). It describes the influence of a particular active strut on the top surface slope:

$$
\Delta S=S^{\prime \prime}-S^{\prime}
$$

- The influence vector $v$ contains the 10 slope variations $\Delta S$ that correspond to the 10 active struts. According to equation (5), its expression is: 


$$
\boldsymbol{v}=\left[\begin{array}{llll}
\Delta S(1) & \Delta S(2) & \cdots & \Delta S(10)
\end{array}\right]^{T}
$$

- The top surface slope after correction $S^{\prime \prime \prime}$ is evaluated when the top surface slope has been corrected through the application of a control command, the vertical coordinate of node $i$ is labeled $z^{\prime \prime \prime}$ and corrected top surface slope $S^{\prime \prime \prime}$ is calculated as follows:

$$
S^{\prime \prime \prime}=\frac{1}{L}\left(z_{43}^{\prime \prime \prime}-\frac{z_{37}^{\prime \prime \prime}+z_{48}^{\prime \prime \prime}}{2}\right)
$$

- The slope compensation SC is defined in order to evaluate top surface slope correction quality using equations (1), (2) and (7). Its expression is:

$$
S C=\frac{S^{\prime}-S^{\prime \prime \prime}}{S^{\prime}-S}
$$

- The transversal slope deviation TSD is introduced in order to evaluate the transversal behavior of the structure (Figure 2). Its expression is:

$$
T S D=\frac{1}{L_{t}}\left[\left(z_{48}^{\prime}-z_{37}^{\prime}\right)-\left(z_{48}-z_{37}\right)\right]
$$

where $L_{t}$ is the transversal distance between nodes 37 and 48 .

- The approximate global stiffness indicator $K$ is evaluated as follows when a point load at node $i$ is labeled $Q_{i}$ :

$$
K=\frac{Q_{37}}{\left|\operatorname{TSSD}\left(Q_{37}\right)\right|}+\frac{Q_{43}}{\left|\operatorname{TSSD}\left(Q_{43}\right)\right|}+\frac{Q_{48}}{\left|\operatorname{TSSD}\left(Q_{48}\right)\right|}
$$

where $Q_{37}, Q_{43}$ and $Q_{48}$ are vertical loads that are applied successively to the structure. $K_{m}$ is measured on the structure and $K_{c}$ is calculated through numerical simulation. 
- The maximal stress ratio $T$ indicates the remaining capacity of the most stressed element. Elements of tensegrity structures carry only normal forces. Struts are in compression and tendons are in tension. The normal force in element $i$ is labeled $N_{i}$ and the capacity of element $i$ is $N_{a d m, i .}$ The maximal stress ratio over all elements $T$ is written as:

$$
T=\max _{i}\left(\frac{N_{i}}{N_{a d m, i}}\right)
$$

Since the structure is not equipped with strain sensors, forces values are determined numerically.

\section{Control framework}

Intelligent-control methodologies such as self-diagnosis, multi-objective shape control and reinforcement learning are organized in a control framework (Figure 3). In practical situations, intelligent structural control involves adaptation in environments that cannot be fully defined. For the purposes of this study, loads are partially defined. A partially-defined load is a known type (for example a single point load) and unknown magnitude and location.

Since loads that are applied to the structure are partially defined, self-diagnosis, Figure 3 right, is required to identify load magnitudes and locations. Control commands are then computed according to self-diagnosis solutions using multi-objective search in order to correct the top-surface-slope deviation due to loads, Figure 3 bottom. Control commands 
are applied to the structure. Reinforcement learning, Figure 3 middle, improves control performance through adapting past experience memorized from previous loading and control events. Details of these steps are described in the following sections.

\section{Self-diagnosis}

Loads are single vertical static loads. Load magnitude and location is unknown. Selfdiagnosis involves magnitude evaluation as well as location and it is carried out using system identification methodology. Similarly to the inverse problem of control command computation presented in (Fest et al. 2004), there is no closed-form solution for selfdiagnosis due to geometric non-linearity. In spite of this, system identification in this study requires no additional measurements.

Comparisons between measured values due to applied loads (subscript $m$ ) and calculated values (subscript $c$ ) of three indicators support system identification. These indicators are top-surface-slope deviation TSSD (Equation 3), transversal-slope deviation TSD (Equation 9) and the influence vector $\boldsymbol{v}$ (Equation 6). Calculated values are determined by dynamic relaxation analysis using kinetic damping. Domer (2003) contains more information related to this methodology. The following steps are carried out:

Step 1: Top surface slope deviation TSSD is the first indicator. The value of $T S S D_{m}$ is measured on the structure. Iteratively for each node of the top surface, load magnitudes are gradually increased and $T S S D_{c}$ is numerically calculated until relation (12) becomes true.

$$
\operatorname{TSSD}_{c}>\mathrm{TSSD}_{m}
$$


Value pairs of load magnitudes and locations create a set of candidate solutions. Negative loads (upwards) are not considered.

Step 2: Transversal-slope deviation TSD is the second indicator. This indicator is used to ensure that trends are the same. This means that candidate solutions that do not satisfy expression (13) are rejected.

$$
\frac{T S D_{c}}{\operatorname{abs}\left(T S D_{c}\right)}=\frac{T S D_{m}}{a b s\left(T S D_{m}\right)}
$$

Experimental measurements show that $2.9 \mathrm{~mm} / 100 \mathrm{~m}$ is an upper bound for precision error of transversal-slope deviation. In situations where transversal-slope deviation is less than $2.9 \mathrm{~mm} / 100 \mathrm{~m}$, no candidate solution is rejected.

Step 3: The influence vector $\boldsymbol{v}$ is the third indicator. Active-control perturbations are applied to evaluate the influence vector $\boldsymbol{v}$. An active-control perturbation is a $1 \mathrm{~mm}$ elongation of an active strut. Among the remaining candidates, the candidate that satisfies expression (14) is taken to be the reference candidate.

$$
\min \left|\boldsymbol{v}_{c}-\boldsymbol{v}_{\boldsymbol{m}}\right|=\min \left(\sqrt{\sum_{j=1}^{10}\left(\Delta S_{c}(j)-\Delta S_{m}(j)\right)^{2}}\right)
$$

where $j$ indicates active strut number. Practical applications of system identification include consideration of errors. An upper bound for the error on slope variations for one single active-control perturbation has been observed to be $e_{p}=0.11 \mathrm{~mm} / 100 \mathrm{~m}$. This error is related to variations in the actuation system and sensor-system accuracy. Candidate solutions that satisfy expression (15) are also taken to be self-diagnosis solutions.

$$
\left|\boldsymbol{v}_{\text {ref }}-\boldsymbol{v}_{c}\right| \leq 10 \cdot e_{p}
$$


where the subscript ref is introduced to define the reference candidate. This process results in a set of candidate solutions for self-diagnosis.

\section{Multi-objective shape control}

As noted in the previous section, there is no closed-form solution for control command computation for shape control of this active tensegrity structure (Figure 1). Multi-objective search is used to select control commands. In practical contexts, active structures need to remain in service as loads change.

While shape control in this study involves maintaining the top surface slope of the structure when subjected to a load, this objective should not be met in isolation. Robustness of both the structure and the active-control system is also important. In this study, robustness of the structure is related to serviceability and safety. Robustness of the active-control system is expressed in terms of active-strut stroke. In situations of active control, robustness is required to accommodate multiple load and control events over service life.

Since the structure is equipped with ten active struts and contains only three measurement points, many combinations of contractions and elongations of active struts satisfy the top surface slope objective to an acceptable degree (Adam and Smith, 2007A). This presents an opportunity to select commands using multi-objective search to control the structure while maintaining robustness. In other words, additional objectives are used to avoid limits of safety and serviceability. The following objectives are relevant: 
- $\quad$ Slope: maintain top-surface slope of the structure,

- $\quad$ Stress: minimize stress-ratio of the most stressed element,

- $\quad$ Stroke: maintain active-strut jacks as close as possible to their midpoint,

- $\quad$ Stiffness: maximize the stiffness.

The two first objectives are related to robustness of the structure. The third objective insures robustness of the active control system. The fourth objective is intended to ensure adequate stiffness of the structure for the next loading event. These four objectives are not complementary. Decision variables are the positions of the ten active struts: $\boldsymbol{x}=\left(x_{1}, x_{2}, \ldots\right.$, $\left.x_{10}\right)$. Quantities and notations that are used to formulate these objective functions are defined in the Section, Terminology and formulas. Objective functions are expressed as follows:

$$
\begin{aligned}
& f_{\text {slope }}=S_{c}^{\prime \prime \prime}(\boldsymbol{x}, \boldsymbol{q})-S_{c} \\
& f_{\text {stress }}=T_{c}(\boldsymbol{x}, \boldsymbol{q}) \\
& f_{\text {stroke }}=\max _{i}\left(x_{i}-x_{i}^{0}\right) \\
& f_{\text {stiffness }}=\frac{1}{K_{c}(\boldsymbol{x}, \boldsymbol{q})}
\end{aligned}
$$

where $\boldsymbol{q}$ describes applied loads. The objective function (16) is the distance between corrected top surface slope and initial slope. Function (17) is the stress ratio of the most stressed element. Function (18) is the maximum stroke among all active struts and function (19) is the inverse of the global stiffness indicator for subsequent loading events. Inequality constraints are intended to prevent failure when the control command is applied. Constraint functions are: 


$$
\begin{aligned}
& g_{\text {no_rupture }}=T_{c}(x, \boldsymbol{q})-1 \leq 0 \\
& g_{\text {no_tension }}=\min _{\text {struts }}\left[N_{c}(\boldsymbol{x}, \boldsymbol{q})\right] \leq 0 \\
& g_{x, \min }=-\left(x_{i}-x_{i}^{\min }\right) \leq 0, \forall i=1, \ldots, 10 \\
& g_{x, \text { max }}=x_{i}-x_{i}^{\max } \leq 0, \forall i=1, \ldots, 10
\end{aligned}
$$

Strut buckling and cable rupture are prevented by constraint (20). Since strut connections are made through contact compression only, tension in struts has to be avoided, Eqn. (21). Stroke constraints, Eqns. (22) and (23), bound active-strut positions. The multi-objective task is represented mathematically as follows:

$$
\begin{array}{ll}
\min & \left\{f_{\text {slope }}(\boldsymbol{x}), f_{\text {stroke }}(\boldsymbol{x}), f_{\text {stress }}(\boldsymbol{x}), f_{\text {stiffness }}(\boldsymbol{x})\right\} \\
\text { subject to: } \quad & g_{\text {no_rupture }}(\boldsymbol{x}, \boldsymbol{q}) \\
& g_{\text {no_tension }}(\boldsymbol{x}, \boldsymbol{q}) \\
& g_{x, \min }(\boldsymbol{x}) \\
& g_{x, \max }(\boldsymbol{x})
\end{array}
$$

The methodology for multi-objective shape control is based on Pareto filtering and hierarchical selection (Pareto 1896). A set of Pareto optimal solutions is built according to the four objectives: slope, Eqn. (16), stress, Eqn. (17), stroke, Eqn. (18) and stiffness, Eqn. (19) and the five constraints: Eqns. (20), (21), (22) and (23). Solutions are generated in order to minimize all objectives. Dominated solutions are rejected. Dominated solutions are defined as solutions that are worse than at least one Pareto optimal solution considering all objectives. 
The selection strategy hierarchically reduces the set of Pareto optimal solutions until one solution remains. This solution for the active strut positions is taken to calculate the control command as follows.

$$
C C=x-x_{0}
$$

where $\boldsymbol{x}_{0}$ is the vector of the initial positions of the ten active struts. The control command is applied to the structure.

\section{Reinforcement learning}

Reinforcement learning is used to improve control performance. Although the proposed learning algorithm employs case-based reasoning, which is often classified as supervised learning, it has evolved into reinforcement learning since it learns from errors in order to support incremental improvements of control commands without an external "teacher". The learning algorithm is divided into memorization, retrieval, adaptation and replacement processes.

After successful self-diagnosis and multi-objective shape control, a case is memorized. A case is composed of the following components: case attributes, control command, slope compensation (Table 1). Case attributes are the response of the structure in terms of top surface slope deviation Eqn. (3), influence vector Eqn. (6) and transversal slope deviation Eqn. (9). The control command is composed of ten active-strut elongations and contractions. The last component is the slope compensation that is measured on the structure when the control command was applied. 
It was observed in the study on self-diagnosis, that different loads of similar magnitudes induce similar responses of the structure according to Eqns. (13) and (15) (Adam and Smith, 2007B). The retrieval function is based on this property. The response of the structure to the current load is evaluated in terms of top surface-slope deviation Eqn. (3), influence vector Eqn. (6) and transversal-slope deviation Eqn. (9). This response is compared with attributes of cases:

$$
\begin{aligned}
& \left|T S S D_{\text {case }}-T S S D_{m}\right| \leq 2.2 \mathrm{~mm} / 100 \mathrm{~m} \\
& \frac{T S D_{\text {case }}}{\left|T S D_{\text {case }}\right|}=\frac{T S D_{m}}{\left|T S D_{m}\right|} \\
& \left|\boldsymbol{v}_{\text {case }}-\boldsymbol{v}_{m}\right|=\sqrt{\sum_{j=1}^{10}\left(\Delta S_{\text {case }}(j)-\Delta S_{m}(j)\right)^{2}} \leq 17 \mathrm{~mm} / 100 \mathrm{~m}
\end{aligned}
$$

where the subscript case refers to case attributes and subscript $m$ to measured values. When conditions Eqns. (25), (26) and (27) are true for a particular case, the behavior of the structure subjected to the current load is said to be similar to this case. This case is retrieved.

Once a case is retrieved, its control command is adapted for shape-control of the structure subjected to current load. Adaptation based on a locally elastic-linear assumption is proposed. The adaptation of control commands is written as follows:

$$
\boldsymbol{C C}=\frac{T S S D_{m}}{T S S D_{\text {case }} \cdot S C_{m}\left(C C_{\text {case }}\right)} \boldsymbol{C C} C_{\text {case }}
$$

Control command adaptation is made over two levels. Control commands are scaled with respect to the ratio $T S S D_{m} / T S S D_{\text {case }}$ and the experimentally observed slope compensation $S C_{m}\left(\boldsymbol{C C}_{\text {case }}\right)$ that was achieved by the control command of the retrieved case. When the 
control command $\boldsymbol{C C}$ is applied, experimentally observed slope compensation $S C_{m}(\boldsymbol{C C})$ is measured. It is rarely equal to $100 \%$. Since this algorithm takes into account $S C_{m}$, it learns from errors. Therefore, it supports command computation through reinforcement learning. In situations where the control command that is adapted from a retrieved case leads to slope compensation improvement, the current case replaces the retrieved case.

\section{Example using the framework}

The implementation of the control framework (Figure 3) on an active tensegrity structure has been validated experimentally over more than fifty load cases. In this paper, adaptation over three load cases: $727 \mathrm{~N}$ at node $16,794 \mathrm{~N}$ at node 16 and $541 \mathrm{~N}$ at node 21 are illustrated. These load cases were selected because they illustrate well the functionality of the framework. Similar quality results were obtained with all other load cases.

A load of $727 \mathrm{~N}$ is applied at node 16 . The top-surface-slope deviation $T S S D_{m}$ that results from this load is measured to be equal to $23 \mathrm{~mm} / 100 \mathrm{~m}$. The transversal-slope deviation $T S D_{m}$ is equal to $-9.1 \mathrm{~mm} / 100 \mathrm{~m}$. Active-control perturbations are applied to the structure. The influence vector $\boldsymbol{v}_{m}$ can be evaluated by measuring slope variations that result from each active-strut elongation (Table 2).

Candidate solutions that induce a top-surface-slope deviation close to the value that is measured on the structure according to Eqn. (12) are listed in Table 3. These six candidates were selected from 15 possibilities. Candidate 1 is not rejected, since its transversal-slope 
deviation is negative according to Eqn. (13). Candidate 2 to 6 are not rejected either, since their transversal-slope deviation is below the error threshold.

The Euclidean distances between influence vectors of the structure and the candidates are listed in Table 4. Since Euclidean distance between influence vectors of the structure and candidate $1,750 \mathrm{~N}$ at node 16 , is minimum, candidate 1 is taken to be the reference candidate according to Eqn. (14). Candidates 2 and 5 are also taken to be self-diagnosis solutions according to Eqn. (15).

Control commands are then computed using multi-objective search, according to each of these three self-diagnosis solutions. The three control commands are listed in Table 5. Control-command computations took approximately 45min using a personal computer (Pentium 4, $3.08 \mathrm{GHz}, 1.00 \mathrm{~GB}$ of RAM). Since the control command that is computed according to candidate $1,750 \mathrm{~N}$ at node 16 , uses the least stroke, it is selected to be applied to the structure.

Figure 4 illustrates the correction of top-surface slope. Top-surface-slope deviation is plotted in the vertical axis versus steps of $1 \mathrm{~mm}$ of active-strut adjustments in the horizontal axis. Zero top-surface-slope deviation means that initial slope is recovered. This control command does not allow the top-surface slope to reach exactly the initial top-surface slope. Slope compensation is equal to $93 \%$. This control event is memorized in the case base. At the end of this control event, the load of $727 \mathrm{~N}$ is removed from node 16 and the active control system is placed in its initial position. 
A load of $794 \mathrm{~N}$ is then applied to the structure, at node 16 . Top-surface-slope is measured on the structure to be equal to $25 \mathrm{~mm} / 100 \mathrm{~m}$. The transversal-slope deviation is equal to $-9.4 \mathrm{~mm} / 100 \mathrm{~m}$. Active-control perturbations are applied to evaluate the influence vector. The influence-vector components are written in Table 6. Since the similarity conditions Eqns. (25), (26) and (27) are satisfied, the case that has been created at the previous control event is retrieved.

$$
\begin{aligned}
& \left|T S S D_{\text {case }}-T S S D_{m}\right|=|23-25|=2 \mathrm{~mm} / 100 \mathrm{~m} \leq 2.2 \mathrm{~mm} / 100 \mathrm{~m} \\
& \frac{T S D_{\text {case }}}{\left|T S D_{\text {case }}\right|}=\frac{T S D_{m}}{\left|T S D_{m}\right|}=-1 \\
& \left|\boldsymbol{v}_{\text {case }}-\boldsymbol{v}_{m}\right|=7.4 \mathrm{~mm} / 100 \mathrm{~m} \leq 17 \mathrm{~mm} / 100 \mathrm{~m}
\end{aligned}
$$

Therefore, the control command that has been used to control the structure when loaded with $727 \mathrm{~N}$ at node 16 is adapted in order to control the structure when loaded with $794 \mathrm{~N}$ at node 16, using Eqn. (28). Adaptation is direct. No iterations are needed. Less than 1s is required for adaptation using the personal computer mentioned above.

$$
\boldsymbol{C C}=\frac{25}{23 \cdot 0.93} \boldsymbol{C} \boldsymbol{C}_{\text {case }}
$$

The command to control the structure subjected to the current load $794 \mathrm{~N}$ at node 16 is written in Table 7. This control command is applied to the structure. Figure 5 illustrates top surface-slope correction. Top-surface-slope deviation is plotted in the vertical axis versus steps of $1 \mathrm{~mm}$ of active-strut adjustments in the horizontal axis. Zero top-surface-slope deviation means that initial slope is recovered. Although the initial top-surface slope is not 
exactly recovered, slope compensation improves with respect to the control event that created the retrieved case. Slope compensation is equal to $101 \%$.

Since slope compensation improves, a new case is created. It replaces the retrieved case. At the end of this control event, the load of $794 \mathrm{~N}$ is removed from node 16 . The active-control system is placed in the initial position.

A load of $541 \mathrm{~N}$ is applied to the structure at node 22. The top-surface-slope deviation is measured to be equal to $144 \mathrm{~mm} / 100 \mathrm{~m}$. The transversal-slope deviation is equal to $2.0 \mathrm{~mm} / 100 \mathrm{~m}$. The active-control perturbations induce the slope variations that are listed in Table 8, in order to create the influence vector.

The candidate solutions that induce a top surface slope that is close (Eqn. 12) to the value measured on the structure are listed in Table 9. Since candidate 3 and 4 have negative transversal-slope deviation, they are rejected according to Eqn. (13). Candidate 1, 650N at node 22, is accepted since its transversal-slope deviation is less then the error threshold. Candidate $3,600 \mathrm{~N}$ at node 50 and $4,850 \mathrm{~N}$ at node 34 , are also accepted since TSD is positive.

Euclidean distances between influence vectors of the structure and candidates are listed in Table 10. Candidate 1, 650N at node 22, is the reference candidate, according to Eqn. (14). No other candidates are accepted, according to Eqn. (15). A control command is computed 
according to this self-diagnosis solution using multi-objective search. This control command is written in Table 11 and applied to the structure.

Figure 6 illustrates top-surface-slope correction. Top surface slope deviation is plotted in the vertical axis versus steps of $1 \mathrm{~mm}$ of active-strut adjustments in the horizontal axis. Zero top-surface-slope deviation means that initial slope is recovered. This control command does not allow the top-surface-slope to reach exactly the initial top-surface slope. Slope compensation is equal to $87 \%$. This control event is memorized in the case base. A new case is created.

To summarize, the control framework was used to control the active tensegrity structure over three loads (Figure 7). When the load of $727 \mathrm{~N}$ was applied to the structure at node 16, the self-diagnosis process was followed by the multi-objective control-command computation taking approximately $45 \mathrm{~min}$. This control command was then applied to the structure. When the load of $794 \mathrm{~N}$ was applied to the structure at node 16 , reinforcement learning was used to retrieve the case that had been created for $727 \mathrm{~N}$ at node 16 and adapt the command that had been computed. The computational time for this adaptation is in the order of milliseconds. The adapted control command was then applied to the structure. When the load of $541 \mathrm{~N}$ was applied to the structure at node 22, the self-diagnosis process was followed by the multi-objective control-command computation. The control command was then applied to the structure. 
Therefore, the control framework provides two possible paths for computation of control commands. The reinforcement-learning path is much faster and improves control-command quality. This path will be taken increasingly as the structure gains experience from previous loading events. Exploratory studies have indicated that, for the loading described in this paper, approximately one hundred and fifty cases would be necessary to ensure that all new loading situations can be controlled through adaptation of cases.

\section{Future work}

Further challenges, such as accommodating more complex loading situations and better case-replacement strategies have been identified. Increased complexity can be accommodated by stochastic-search strategies such as those used to search for good control commands (Domer et al 2003). There are also several other opportunities for improvements in self-diagnosis, multi-objective control, self-repair and reinforcement learning. New results are expected to provide further progress in the field of adaptive civil-engineering structures.

Since tensegrities are lightweight structures, they are susceptible to vibrations. In practical situations, civil-engineering structures are subjected to mechanical, pedestrian, traffic, wind and earthquake loading and in addition, structures in space could be subjected to rapid temperature changes and impact due to debris. In situations where an excitation frequency is close to natural frequencies, resonance could damage the structure. Current work includes a study of the effect of vibrations and the capacity of the active-control system to attenuate them. 


\section{Conclusions}

A control framework that integrates self-diagnosis, multi-objective shape control and reinforcement learning contributes to the advancement of experimental and analytical knowledge of adaptive civil-engineering structures.

Self-diagnosis extends active control to adaptation in partially defined environments. The control system of an active tensegrity structure can be used to support self-diagnosis. Multiobjective search has been used to select control commands that allow for shape control of an active tensegrity structure while maintaining robustness of the structure and its control system. An algorithm for reinforcement learning is proposed to improve active control. Reinforcement learning helps improve the control of an active tensegrity structure with respect to command-computation time and control quality. More generally, the combination of learning algorithms with active-control systems is attractive for improving the usefulness of structural control.

The control framework that is presented in this document has the potential to be adapted to more complex loading and damage as well as to more complex active structures in order to create a wider range of adaptive civil-engineering structures.

\section{Acknowledgments}

The authors would like to thank the Swiss National Foundation for supporting this work through funding contract 2000-067740. K. Shea, R. Motro, T. Keller, B. Domer, Y. RobertNicoud, P. Kripakaran, S. Saitta and H. Pelletier are recognized for discussions and advice. 
E. Fest built the structure and the control system. B. Domer subsequently improved control and implemented the case-based reasoning study. B. Raphael provided support during programming of the control system. We are also grateful to Passera \& Pedretti SA (Lugano, Switzerland), Lust-Tec GmbH (Zürich, Switzerland), S. Demierre and P. Gallay for their contributions. 


\section{Appendix}

$\begin{array}{ll}K & \text { Approximate global-stiffness indicator } \\ S & \text { Top-surface slope of the structure at initial state } \\ S^{\prime} & \text { Top-surface slope after loading } \\ S^{\prime \prime} & \text { Top-surface slope after perturbation } \\ S^{\prime \prime \prime} & \text { Top-surface slope after correction } \\ S C & \text { Slope compensation } \\ T & \text { Maximum stress-ratio } \\ T S D & \text { Transversal-slope deviation } \\ T S S D & \text { The top-surface-slope deviation } \\ \Delta S & \text { Slope variation } \\ v & \text { Influence vector }\end{array}$




\section{References}

Adam, B. and Smith, I.F.C., (2007A), “Tensegrity Active Control: a Multi-Objective Approach”, Journal of Computing in Civil Engineering, Vol 21, No 1, pp 3-10.

Adam, B. and Smith, I.F.C., (2007B) "Self-diagnosis and Self-repair of an Active Tensegrity Structure”, Journal of Structural Engineering, Vol 133, 2007, pp 1752-1761.

Adam, B. and Smith, I.F.C., (2008), “Reinforcement Learning for Structural Control”, Journal of Computing in Civil Engineering, Vol 22, No 2.

Averseng, J., and Crosnier, B. (2004). "Static and Dynamic Robust Control of Tensegrity Systems”, Journal of The International Association for Shell and Spatial Structures, 45, 169-174.

Ben Kahla, N., and Moussa, B. (2002), “Effect of a Cable Rupture on Tensegrity Systems”, International Journal of Space Structures, 17(1), 51-65.

Denning, P.J. (1976). “Fault Tolerant Operating Systems”, ACM Computing survey, 8(4), 359-389.

Djouadi, S., Motro, R., Pons, J.C., and Crosnier, B. (1998). “Active Control of Tensegrity Systems”, Journal of Aerospace Engineering, 11, 37-44. 
Domer, B., (2003). "Performance enhancement of active structures during service lives", Thèse no 2750, Ecole Polytechnique Fédérale de Lausanne.

Domer, B., Raphael, B., Shea, K. and Smith, I.F.C. (2003), “A study of two stochastic search methods for structural control”, Journal of Computing in Civil Engineering, 17(3), 132-141.

Domer, B., and Smith, I.F.C. (2005). “An Active Structure that learns”, Journal of Computing in Civil Engineering, 19(1), 16-24.

Fest, E. (2002). "Une structure active de type tenségrité”, Thèse no 2701, Ecole Polytechnique Fédérale de Lausanne.

Fest, E., Shea, K., Domer, B., and Smith, I.F.C. (2003). “Adjustable Tensegrity Structure”, Journal of Structural Engineering, 129(4), 515-526.

Fest, E., Shea, K., and Smith, I.F.C. (2004). “Active Tensegrity Structure”, Journal of Structural Engineering, 130(10), 1454-1465.

Floreano, D., and Mondada, F. (1998). "Evolutionary neurocontrollers for autonomous mobile robots”, Neural Networks, (11), 1461-1478. 
Fu, F. (2005). "Structural behavior and design methods of Tensegrity domes", Journal of Constructional Steel Research, 61, 23-35.

Kanchanasaratool, N., and Williamson, D. (2002). "Modelling and control of class NSP tensegrity structures”, International Journal of Control, 75(2), 123-139.

Kawaguchi, K., Pellegrino, S., and Furuya, H. (1996). “Shape and Stress Control Analysis of Prestressed Truss Structures”, Journal of Reinforced Plastics and Composites, 15, 12261236.

Lazopoulos, K.A. (2005). "Stability of an elastic cytoskeletal tensegrity model”, International Journal of Solids and Structures, 42, 3459-3459.

Motro, R., (1984), "Forms and forces in tensegrity systems", In:Nooshil, H. (Ed.), Proceedings of Third International Conference on Space Structures. Elsevier, Amsterdam, 180-185.

Motro, R. (2003). “Tensegrity: Structural systems for the future”, Hermes, ed., Penton Science, U.K., 280.

Noak, T., Ruth, J., and Müller, U. (2006). “Adaptive Hybrid Structures”, Proceedings of the International Conference on Adaptable Building Structures, Adaptables2006, Eindhoven, The Netherlands, 03-05 July 2006. 
Pawlowski, P., and Holnicki-Szulc, J. (2004). “Adaptive Structures under Extreme Loads Impact detection, Self-adaptation, Self-repairing”, Proceedings of the Third European Conference on Structural Control, Vienna, Austria.

Raphael, B., and Smith, I.F.C. (2003). “A direct stochastic algorithm for global search”, J of Applied Mathematics and Computation, 146(2-3), 729-758.

Shea, K., and Smith, I.F.C. (1998). “Intelligent structures: A new direction in structural control”, Artificial Intelligence in Structural Engineering, Computer Science, LNAI 1454, Springer, Heidelberg, pp 398-410.

Shea, K., Fest, E., and Smith, I.F.C. (2002). “Developing intelligent tensegrity structures with stochastic search”, Advanced Engineering Informatics, 16, 21-40

Skelton, R.E., Helton, J.W., Adhikari, R., Pinaud, J.P., and Chan, W. (2000). “An introduction to the mechanics of tensegrity structures”, Handbook on mechanical systems design, CRC, Boca Raton, Fla.

Sobeck, W., Teuffel, P., Weilandt, A., and Lemaitre, C. (2006). “Adaptive and Lightweight”, Proceedings of the International Conference on Adaptable Building Structures, Adaptables2006, Eindhoven, The Netherlands, 03-05 July 2006. 
Soong, T.T., and Manolis, G.D. (1987). “Active structures”, Journal of structural engineering, 113(11), 2290-2301.

Sultan, C. (1999). "Modeling, design and control of tensegrity structures with applications”, PhD thesis, Purdue Univ., West Lafayette, Ind.

Sultan, C., and Skelton, R. (2003). "Deployment of tensegrity structures”, International Journal of Solids and Structures, 40, 4637-4657.

Teuscher, C., Mange, D., Stauffer, A., and Tempesti, G. (2003). "Bio-inspired computing tissues: towards machine that evolve, grow, and learn”, BioSystems, 68, 235-244.

Van de Wijdeven, J. and de Jager, B., (2005), “Shape Change of Tensegrity Structures: Design and Control”, Proceedings of the American Control Conference, Protland, OR, USA, 2522-2527.

Von Neumann, J. (1966), “The theory of Self-Reproducing Automata” University of Illinois Press, Urbana.

Wada, B.K., and Das, S. (1991). Application of adaptive structure concepts to civil structures, Intelligent structures-monitoring and control, Amsterdam, Elsevier, 195-217 
Williamson, D., Skelton, R.E. and Han, J., (2003), “Equilibrium conditions of a tensegrity structure”, International Journal of Solids and Structures, 40, 6347-6367.

Yao, J.T.P. (1972). “Concept of structural control”, ASCE J. Struct. Div., 98, 1567-1574.

Zuk, W. (1968). “Kinetic Architecture”, Civil Engineering, ASCE, December, 62-62.

This work is licensed under a Creative Commons Attribution-NonCommercial-

\section{(c) $($ i) $(9)$}

NoDerivatives 4.0 International License 


\section{Figures}

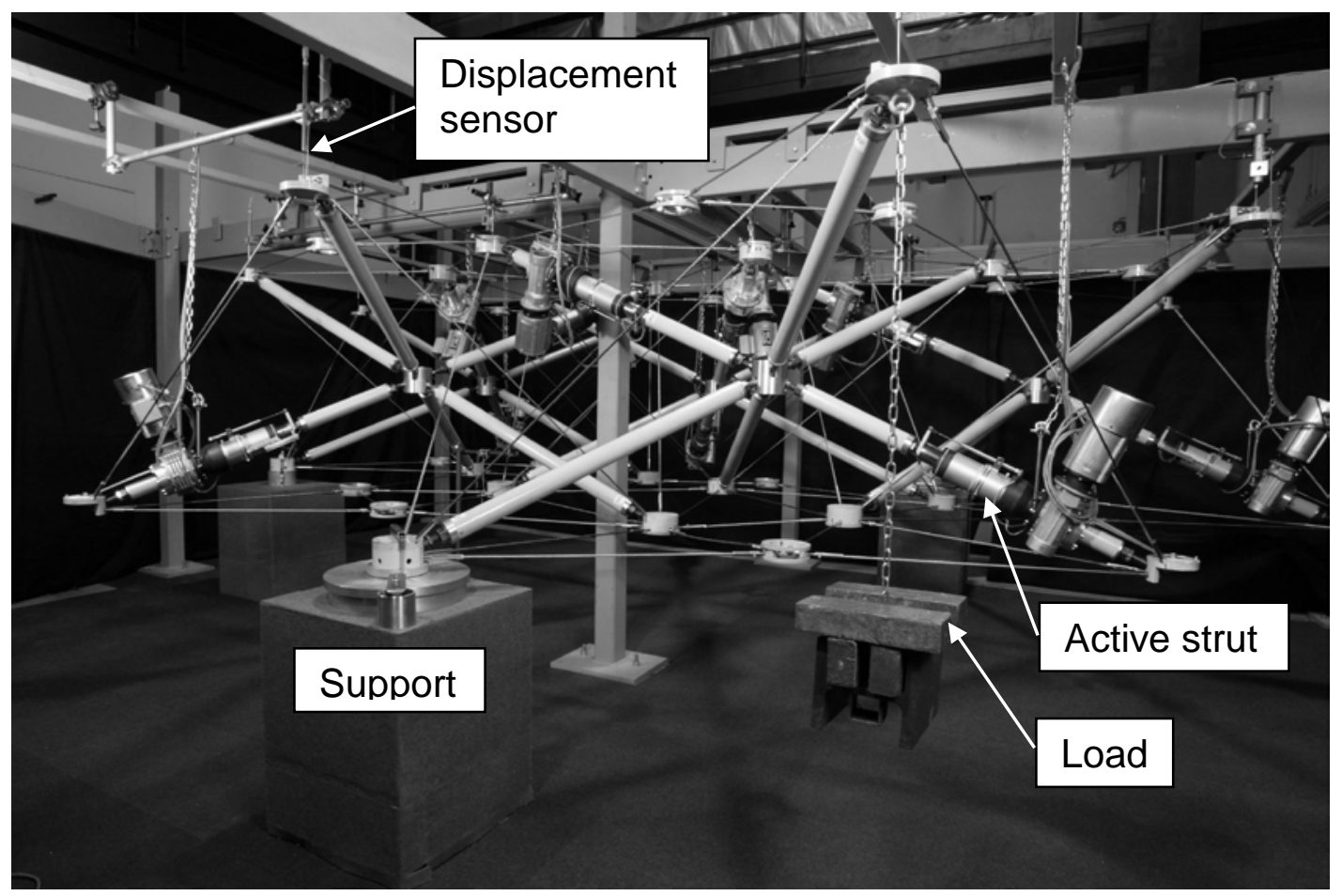

Fig. 1. Five-module tensegrity structure, $15 \mathrm{~m}^{2}$ ground projection, 10 active struts, 3 displacement sensors 


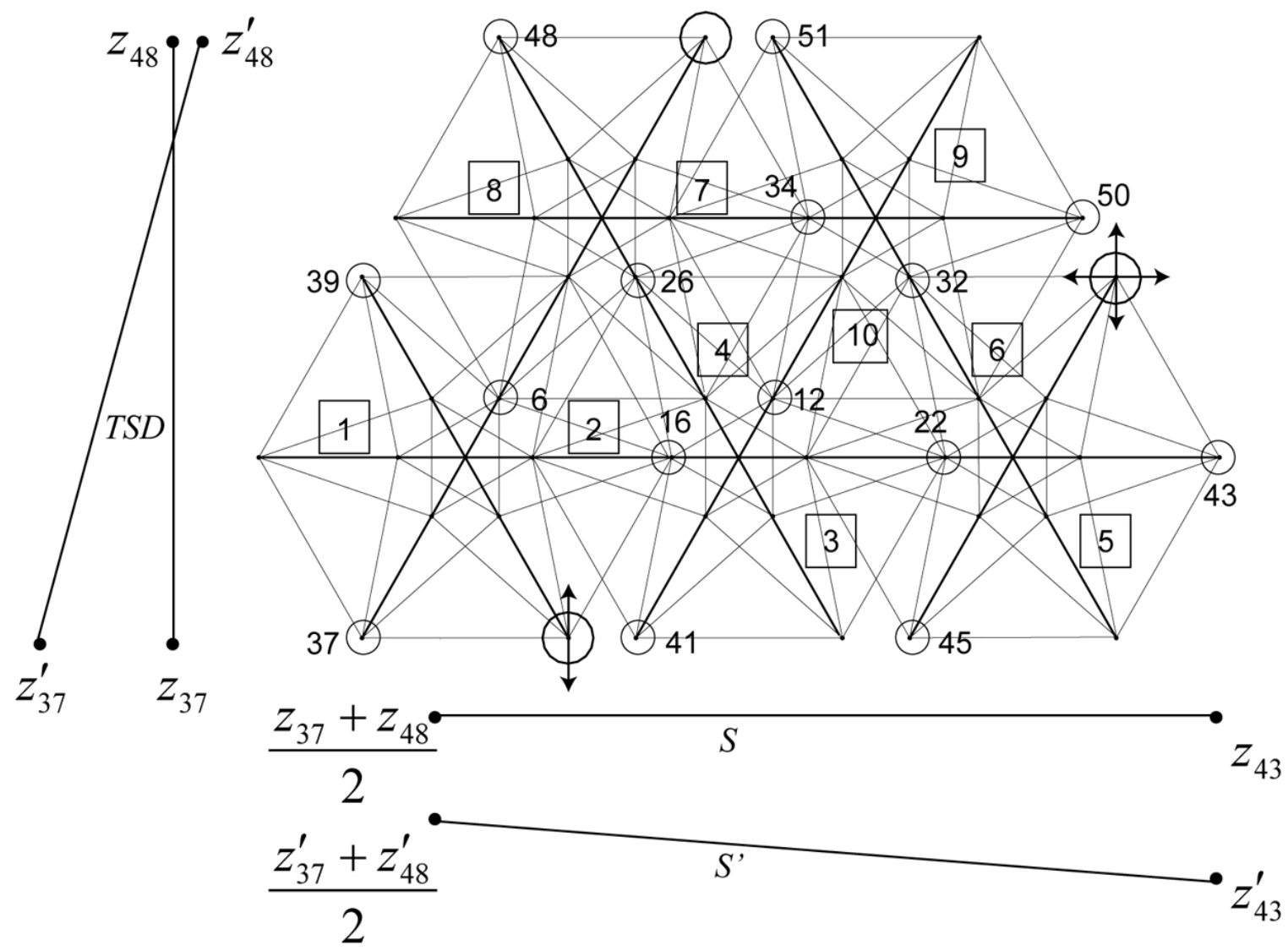

$\uparrow \begin{aligned} & \text { Support conditions } \\ & \text { Circle: vertical restraint } \\ & \text { Arrows: horizontal restraint in the direction of the arrows }\end{aligned}$

Fig. 2. View from the top of the active tensegrity structure 


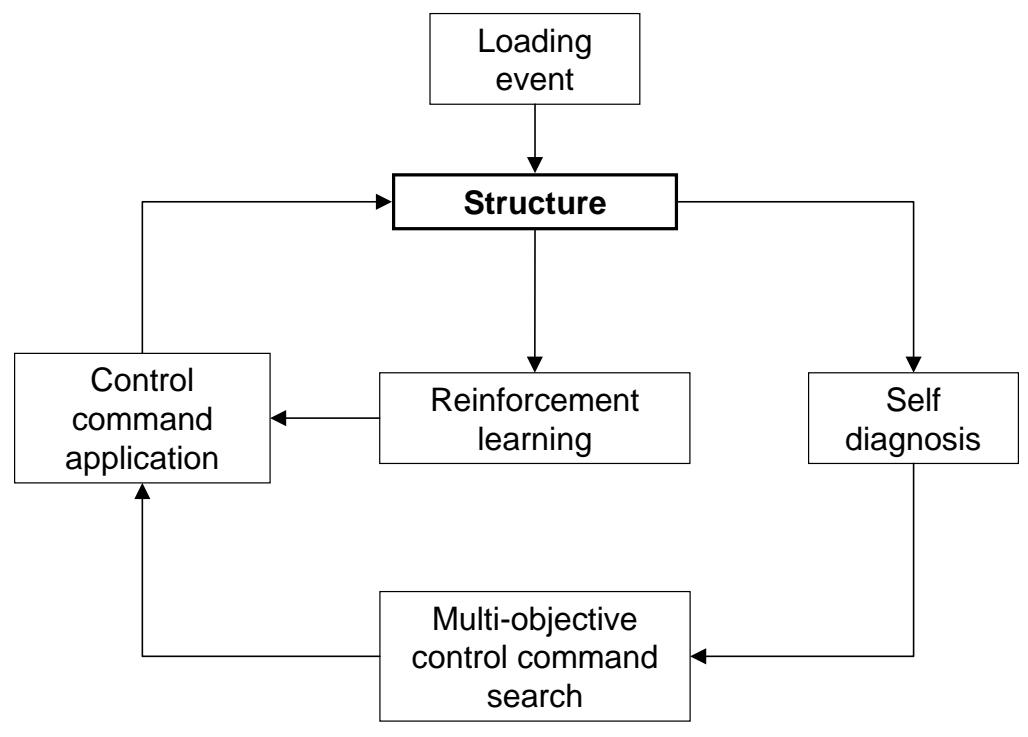

Figure 3. Control framework 


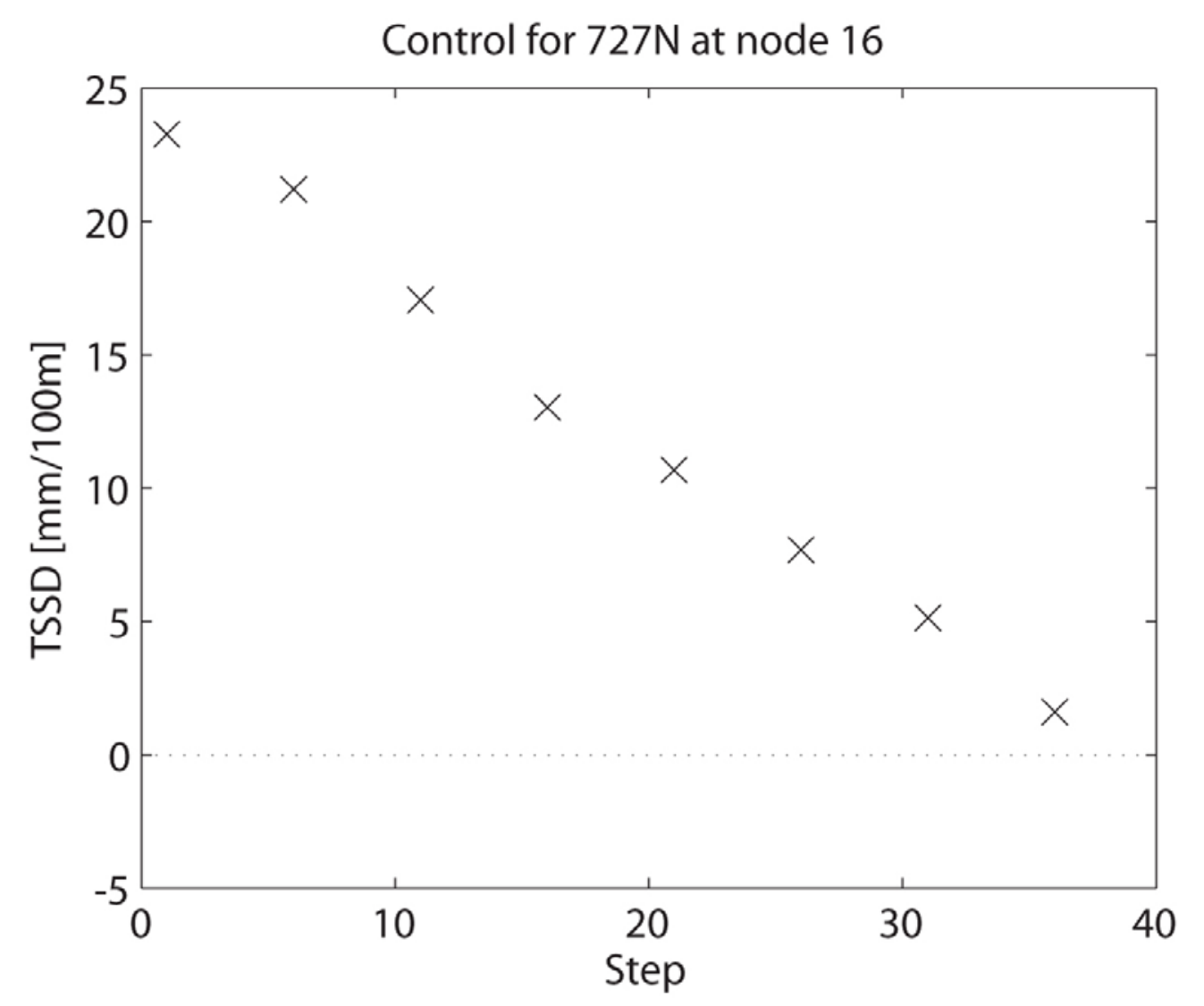

Figure 4. Correction of top surface slope 


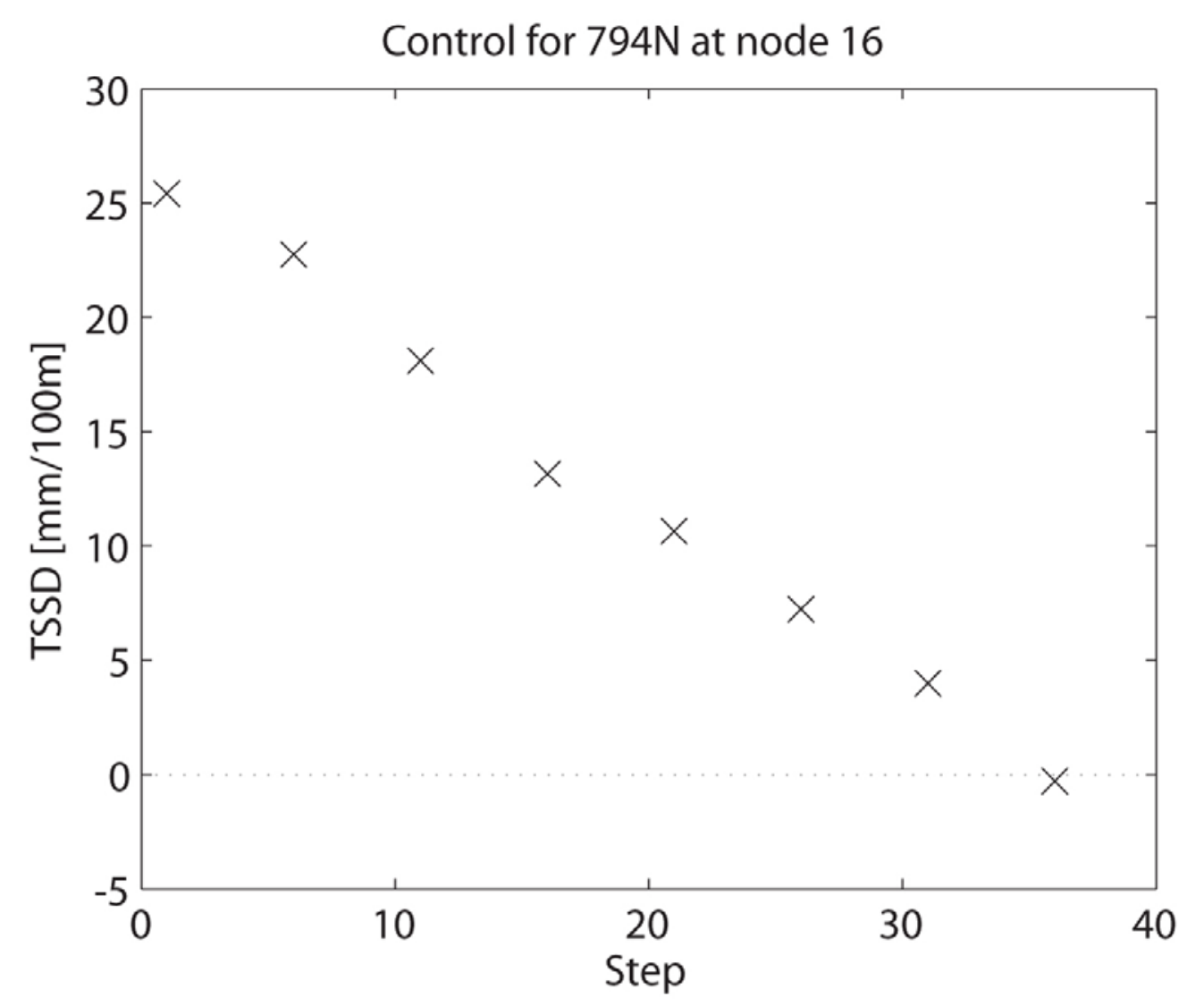

Figure 5. Correction of top surface slope 


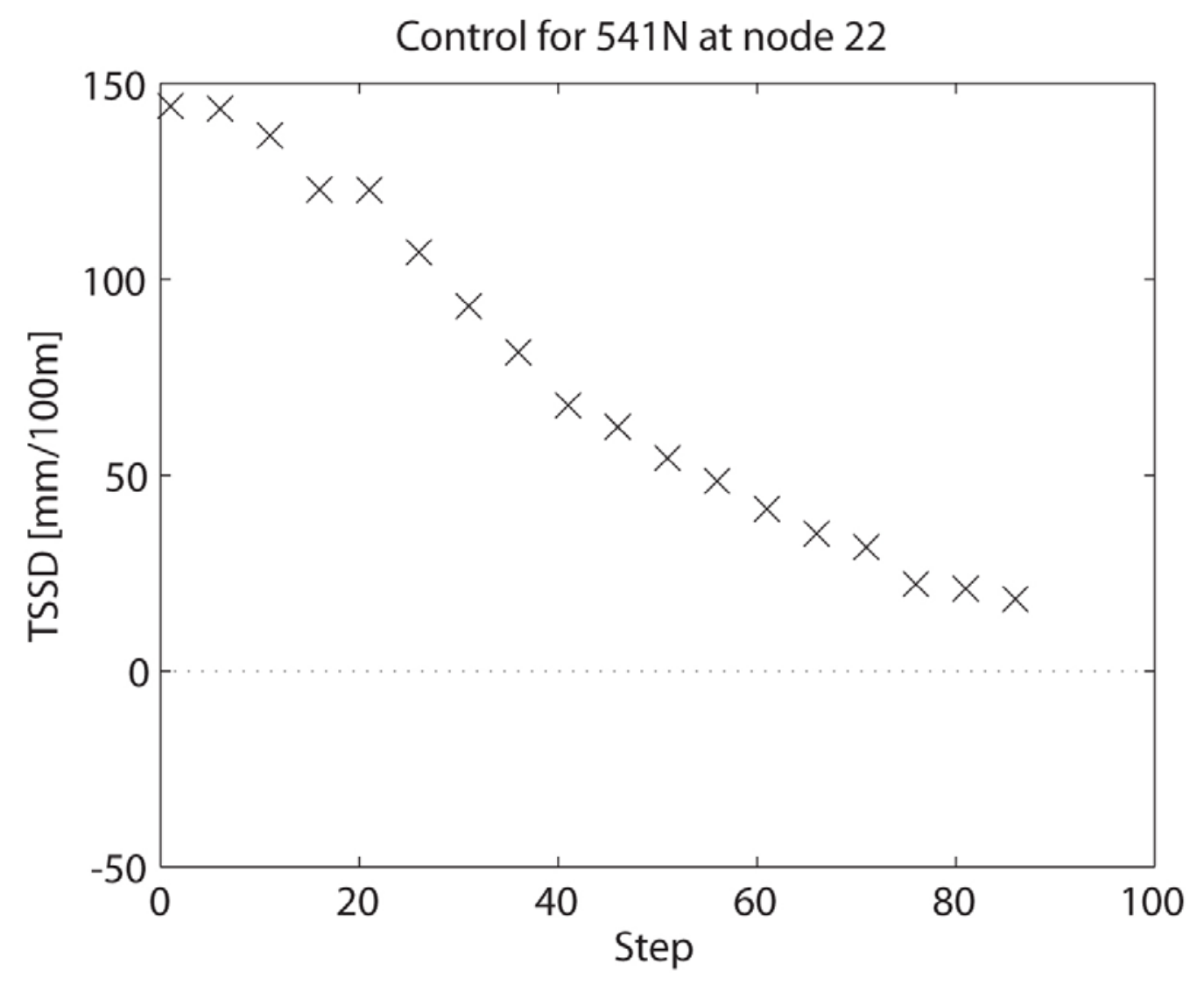

Figure 6. Correction of top surface slope 


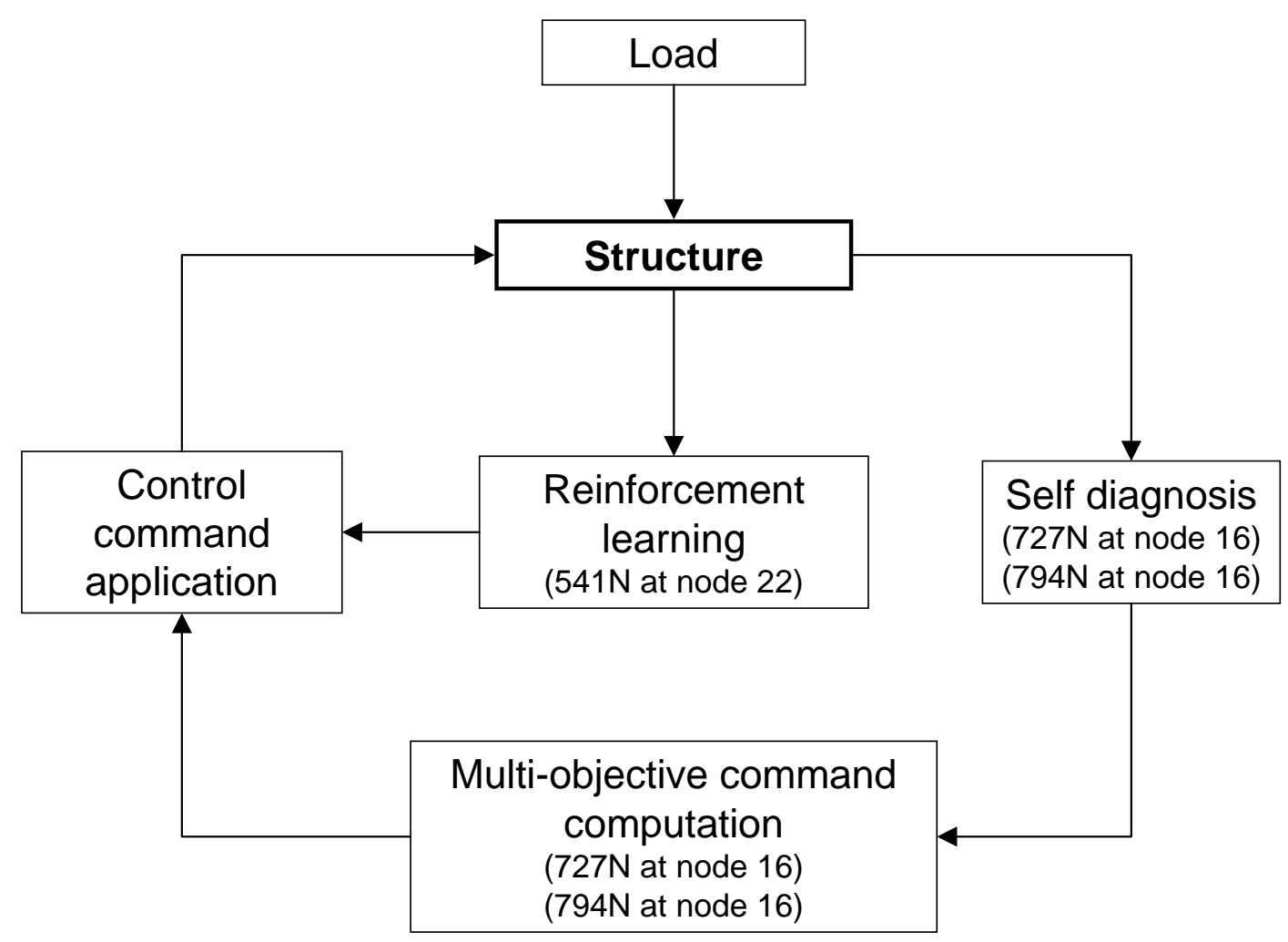

Figure 7. Summary of using the control framework for three loading events 


\section{Tables}

Table 1. Components of a case

Response of the structure Control command

$T S S D_{\text {case }}, v_{\text {case }}, T S D_{\text {case, }}$
CC $_{\text {case }}$
Slope compensation

$S C_{m}\left(\boldsymbol{x}_{\text {case }}\right)$ 
Table 2. Slope variations measured on the structure

\begin{tabular}{ccccccccccc} 
Active strut & $\mathbf{1}$ & $\mathbf{2}$ & $\mathbf{3}$ & $\mathbf{4}$ & $\mathbf{5}$ & $\mathbf{6}$ & $\mathbf{7}$ & $\mathbf{8}$ & $\mathbf{9}$ & $\mathbf{1 0}$ \\
\hline $\boldsymbol{\Delta S}[\mathbf{m m} / \mathbf{1 0 0 m}]$ & 17.0 & 21.1 & -19.2 & -15.1 & -31.9 & -30.9 & 25.7 & 26.9 & -7.4 & -7.0
\end{tabular}


Table 3. Candidate solutions

\begin{tabular}{|c|c|c|c|c|}
\hline Candidate & Node & Magnitude [N] & TSSD $_{c}[\mathrm{~mm} / \mathbf{1 0 0 m}]$ & $\mathrm{TSD}_{\mathrm{c}}[\mathrm{mm} / \mathbf{1 0 0 m}]$ \\
\hline 1 & 16 & -750 & 24.0 & -3.6 \\
\hline 2 & 22 & -100 & 24.6 & -0.2 \\
\hline 3 & 45 & -950 & 24.4 & -1.7 \\
\hline 4 & 43 & -100 & 26.7 & -0.9 \\
\hline 5 & 50 & -100 & 25.7 & 1.1 \\
\hline 6 & 34 & -150 & 26.3 & 1.4 \\
\hline
\end{tabular}


Table 4. Euclidean distances between influence vectors of structure and candidates

\begin{tabular}{ccccccc} 
Candidate & $\mathbf{1}$ & $\mathbf{2}$ & $\mathbf{3}$ & $\mathbf{4}$ & $\mathbf{5}$ & $\mathbf{6}$ \\
\hline$\left|\boldsymbol{v}_{c}-\boldsymbol{v}_{m}\right|$ & 21.0 & 22.0 & 42.4 & 22.5 & 21.9 & 23.0
\end{tabular}


Table 5. Control commands according to the three self-diagnosis solutions

\begin{tabular}{ccccccccccc} 
& \multicolumn{10}{c}{ Control command [mm] } \\
\cline { 2 - 12 } Candidate & $\mathbf{1}$ & $\mathbf{2}$ & $\mathbf{3}$ & $\mathbf{4}$ & $\mathbf{5}$ & $\mathbf{6}$ & $\mathbf{7}$ & $\mathbf{8}$ & $\mathbf{9}$ & $\mathbf{1 0}$ \\
\hline $\mathbf{1}$ & 0.4 & 0.4 & -0.4 & 0.0 & -0.4 & -0.4 & 0.3 & 0.4 & 0.0 & 0.0 \\
$\mathbf{2}$ & 0.5 & 0.4 & -0.5 & 0.1 & -0.2 & -0.2 & 0.4 & 0.5 & 0.3 & 0.5 \\
$\mathbf{5}$ & 0.4 & 0.4 & -0.1 & -0.2 & -0.2 & -0.3 & 0.5 & 0.3 & 0.2 & 0.2
\end{tabular}


Table 6. Slope variations measured on the structure

\begin{tabular}{ccccccccccc} 
Active strut & $\mathbf{1}$ & $\mathbf{2}$ & $\mathbf{3}$ & $\mathbf{4}$ & $\mathbf{5}$ & $\mathbf{6}$ & $\mathbf{7}$ & $\mathbf{8}$ & $\mathbf{9}$ & $\mathbf{1 0}$ \\
\hline $\boldsymbol{\Delta S}[\mathbf{m m} / \mathbf{1 0 0 m}]$ & 18.2 & 21.1 & -17.0 & -14.6 & -30.9 & -29.5 & 25.4 & 26.8 & -7.0 & -6.5
\end{tabular}


Table 7. Control commands adapted from retrieved case

\begin{tabular}{cccccccccc}
\multicolumn{10}{c}{ Control command [mm] } \\
\hline $\mathbf{1}$ & $\mathbf{2}$ & $\mathbf{3}$ & $\mathbf{4}$ & $\mathbf{5}$ & $\mathbf{6}$ & $\mathbf{7}$ & $\mathbf{8}$ & $\mathbf{9}$ & $\mathbf{1 0}$ \\
\hline 0.4 & 0.5 & -0.1 & -0.3 & -0.3 & -0.3 & 0.6 & 0.3 & 0.2 & 0.3
\end{tabular}


Table 8. Slope variations measured on the structure

\begin{tabular}{ccccccccccc} 
Active strut & $\mathbf{1}$ & $\mathbf{2}$ & $\mathbf{3}$ & $\mathbf{4}$ & $\mathbf{5}$ & $\mathbf{6}$ & $\mathbf{7}$ & $\mathbf{8}$ & $\mathbf{9}$ & $\mathbf{1 0}$ \\
\hline $\boldsymbol{\Delta S}[\mathbf{m m} / \mathbf{1 0 0 m}]$ & 22.0 & 23.3 & -13.7 & -10.3 & -23.7 & -21.1 & 25.6 & 26.4 & -3.8 & -3.6
\end{tabular}


Table 9. Candidate solutions

\begin{tabular}{ccccc} 
Candidate & Node & Magnitude [N] & TSSD $_{\boldsymbol{c}}[\mathbf{m m} / \mathbf{1 0 0 m}]$ & TSD $_{\boldsymbol{c}}[\mathbf{m m} / \mathbf{1 0 0 m}]$ \\
\hline 1 & 16 & -750 & -24.0 & -3.6 \\
2 & 22 & -100 & -24.6 & -0.2 \\
3 & 45 & -950 & -24.4 & -1.7 \\
4 & 43 & -100 & -26.7 & -0.9
\end{tabular}


Table 10. Euclidean distances between influence vectors of structure and candidates

\begin{tabular}{cccc} 
Candidate & $\mathbf{1}$ & $\mathbf{2}$ & $\mathbf{3}$ \\
\hline$\left|\boldsymbol{v}_{c}-\boldsymbol{v}_{m}\right|$ & 20.6 & 21.8 & 28.7
\end{tabular}


Table 11. Control commands [mm] adapted from retrieved case

Active strut

\begin{tabular}{cccccccccc}
\hline $\mathbf{1}$ & $\mathbf{2}$ & $\mathbf{3}$ & $\mathbf{4}$ & $\mathbf{5}$ & $\mathbf{6}$ & $\mathbf{7}$ & $\mathbf{8}$ & $\mathbf{9}$ & $\mathbf{1 0}$ \\
\hline 3.0 & 0.7 & 1.3 & -1.8 & -3.0 & -3.0 & 2.0 & 1.5 & -1.1 & -1.4
\end{tabular}




\section{Figure captions}

Fig. 1. Five-module tensegrity structure, $15 \mathrm{~m}^{2}$ ground projection, 10 active struts, 3 displacement sensors

Fig. 2. View from the top of the active tensegrity structure

Fig. 3. Closed control-loop: methodologies

Fig. 4. Correction of top surface slope

Fig.5. Correction of top surface slope

Fig 6. Correction of top surface slope 


\section{Table captions}

Table 1. Loading situations for self-diagnosis experimental testing

Table 2. Summary of self-diagnosis solutions for the 11 load situations presented in Table

Table 3. Damage location solutions for cable 7, 121 and 128 broken

Table 4. Scenario of multiple loading events that are successively applied to the structure

Table 5. Slope compensation $\mathrm{SC}_{\mathrm{m}}$ when the structure is controlled with control commands using self-diagnosis solutions as input 